\title{
An Early Warning Influenza Model using Alberta Real- Time Syndromic Data (ARTSSN)
}

\author{
Paul Smetanin'1, Rita K. Biel², David Stiff', Douglas McNeil', Lawrence Svenson ${ }^{3}$, Hussain \\ R. Usman ${ }^{2}$, David P. Meurer ${ }^{2}$, Jane Huang², Vanessa Nardelli², Christopher Sikora² and \\ James Talbot $^{3}$
}

${ }^{1}$ RiskAnalytica, Toronto, ON, Canada; ${ }^{2} \mathrm{AHS}$, Calgary, AB, Canada; ${ }^{3} \mathrm{AH}$, Edmonton, $\mathrm{AB}$, Canada

\section{Objective}

We developed early warning algorithms using data from ARTSSN and used them to detect signatures of potential pandemics and provide regular weekly forecasts on influenza trends in Alberta during 2012-2014

\section{Introduction}

Standardized electronic pre-diagnostic information is routinely collected in Alberta, Canada. ARTSSN is an automated real-time surveillance data repository able to rapidly refresh data that include school absenteeism information, calls about health concerns from Health Link Alberta (HLA); a provincial telephone service for health advice and information, and emergency department (ED) visits categorized by standardized chief complaint $(\mathrm{CC})^{1}$. Until recently, real-time ARTSSN data for public health surveillance and decision making has been underutilized.

\section{Methods}

A two-part Alberta Influenza Model was constructed using a Bayesian approach and historic HLA and Edmonton ED data from ARTSSN: an agent-based, event-driven infectious disease model simulating the spread of influenza in the province and a simulation module to model the events that would be recorded in ARTSSN due to influenza circulating in the population. During 2012-2013 and 2013-2014, the algorithms were used to provide weekly updates of the predicted attack rate and influenza season peak time to a provincial surveillance team. Syndromic indicators of influenza-like-illness (ILI) were selected from HLA protocols and ED CCs, compared against the Alberta Health influenza case definition ${ }^{2}$, and included in the model. In 2013-2014, additional CCs were included if an ILI+ screen was recorded. The model was implemented to detect abnormal events, such as higher than normal attack rates or atypical peak times. A prior distribution assumption based on historical data was used in the analysis; attack rate of $12.5 \%$, peak time of February 1 and a pandemic probability of once every 30 years.

\section{Results}

A test of the model using simulated and historical H1N1 pandemic observations showed that the early warning algorithms effectively distinguished pandemics from seasonal influenza well before the peak. No pandemic triggering occurred during the two influenza seasons 2012-2014, suggesting that this tool can be effective in pandemic preparedness. Based on lab confirmed cases, the influenza peak in Alberta actually occurred during influenza reporting week 1 (Dec 30-Jan 5) in 2012-2013 and week 2 (Jan 5-10) in 2013-2014. During the 2012-2013 pilot season, the model predicted the peak time within 1 week of the true peak as early as October 25. During 20132014 , the model predicted the peak within 2 weeks of the true peak as early as November 4 . The final model estimates showed that 20122013 was a typical influenza season with an expected attack rate of $11.8 \%( \pm 3.2 \%)$ and a peak in early January (Jan $6 \pm 27$ days). 20132014 was similar with a final attack rate of $11.5 \%( \pm 3.1 \%)$ and a peak time of mid-January (Jan $10 \pm 27$ days). The early estimates of peak time were in line with what other trending tools such as FluWatch and Google flu trends revealed ${ }^{3,4}$. The forecasts of influenza attack rates and peak times were used by decision makers to guide allocation and efficient use of resources, such as acquisition of additional vaccine or decisions about opening a rapid assessment centre, and public communications. The modelling and forecasts heightened awareness and discussion among medical officers, surveillance and public health staff, as health resource management decisions were made.

\section{Conclusions}

The predictive model developed using real-time ARTSSN data, used prospectively, is a promising tool for influenza planning and preparedness.

\section{Keywords}

influenza; syndromic surveillance; predictive model; pandemic; influenza peak time

\section{Acknowledgments}

We thank Bonita Lee, Kevin Fonseca, Albert de Villiers, Sandra Marini, Richard Golonka, Linda Duffley, Steven Probert, Elizabeth Henderson, Evan Jones, Marcia Johnson, Gerry Predy, Carla Briante, Lynette Katsivo, Alysha Visram, Annette Lemire and Bryan Wicentowich.

\section{References}

1. Fan S, Blair C, Brown A, et al. A multi-function public health surveillance system and the lessons learned in its development: the Alberta real-time syndromic surveillance net. Can J Pub Health 2010; 101(6):454-8.

2. Alberta Health Influenza Case Definition. Alberta Health Public Health Notifiable Disease Management Guidelines, October 2012. http:// www.health.alberta.ca/documents/Guidelines-Influenza-2012.pdf.

3. FluWatch http://www.phac-aspc.gc.ca/fluwatch/13-14/w02_14/indexeng.php.

4. Google flu trends http://www.google.org/flutrends/ca/\#CA-AB.

\section{${ }^{*}$ Rita K. Biel}

E-mail: rita.biel@albertahealthservices.ca 